\title{
Electron Transfer Processes in Ferrocene-Modified Poly(ethylene glycol) Monolayers on Electrodes
}

\author{
Tom Steentjes, Pascal Jonkheijm,*i[ and Jurriaan Huskens**i]
}

Molecular Nanofabrication Group, MESA + Institute for Nanotechnology, University of Twente, P.O. Box 217, 7500 AE Enschede, The Netherlands

\section{Supporting Information}

ABSTRACT: Electrochemistry is a powerful tool to study selfassembled monolayers. Here, we modified cystamine-functionalized electrodes with different lengths of linear poly(ethylene glycol) (PEG) polymers end-functionalized with a redox-active ferrocene (Fc) group. The electron transport properties of the Fc probes were studied using cyclic voltammetry. The Fc moiety attached to the shortest PEG $\left(M_{\mathrm{n}}=250 \mathrm{Da}\right)$ behaved as a surface-confined species, and the homogeneous electron transfer rate constants were determined. The electron transfer of the ferrocene group on the longer PEGs $\left(M_{\mathrm{n}}=3.4,5\right.$, and $\left.10 \mathrm{kDa}\right)$ was shown to be driven by diffusion. For low surface densities, where the polymer exists in the mushroom conformation, the diffusion coefficients $(D)$ and rate constants were increasing with polymer length. In the loose brush conformation, where the polymers are close enough to interact with each other, the thickness of the layers $(e)$ was unknown and a parameter $D^{1 / 2} / e$ was determined. This parameter showed no dependence on surface density and an increase with polymer length.
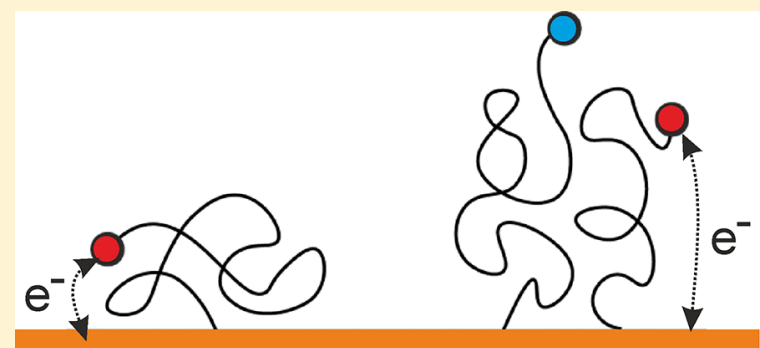

\section{INTRODUCTION}

Understanding conformations of (bio)polymers at surfaces is important since such layers are widely used for electrochemical detection methods, for example, for DNA sensing. ${ }^{1-7}$ The mechanism of so-called E-DNA sensors relies on changes in conformation upon binding with the analyte, and this conformational change results in a change in signal. ${ }^{3}$ As such, the flexibility of the linker plays an important role in the signal output.

The configuration of end-tethered polymers on surfaces is dictated by the surface density and the length of the polymers. ${ }^{8,9}$ The transition between the mushroom and brush conformation, where the polymers begin to overlap, has been modeled $^{10,11}$ and observed experimentally ${ }^{12,13}$ with different results for the onset of the brush formation. If the molecules are tethered with an electrochemical probe, the behavior of the end-group can be studied using electrochemical methods. Several studies have been performed with poly(ethylene glycol) (PEG) layers end-tethered with ferrocene, both with an atomic force/scanning electrochemical microscope setup $^{14}$ and on single electrodes. ${ }^{15,16}$ The polymer behavior in the mushroom configuration, where the polymer chains exist as isolated entities, has been modeled. ${ }^{17}$ These studies have shown that the electrochemical response of the end-group involves diffusion to the surface. As the surface density is increased, which brings about conformational changes, changes in bound diffusion and kinetics can be expected, as have indeed been observed for DNA and PNA layers. ${ }^{18}$ A detailed experimental study on a well-defined model system in which the length and density of surface-attached PEG chains are varied is however currently lacking.

Herein we study the effect of polymer length and surface density of end-tethered PEG layers immobilized on electrodes, in order to study the electron transfer behavior of the endgroup. The surface densities are varied to achieve the mushroom and loose brush conformations, and the influence of the varying surface density on the electrochemical properties of the ferrocene group is established using cyclic voltammetry.

\section{RESULTS AND DISCUSSION}

PEG chains bearing on one end a ferrocene $(\mathrm{Fc})$ moiety, used for electrochemical detection, and on the other end a reactive succinimide (NHS) group, used for surface attachment, were synthesized according to known procedures. ${ }^{15,19}$ Fc-PEG-NHS derivatives of four molecular weights $\left(M_{n}=0.25,3.4,5\right.$, and 10 $\mathrm{kDa}$, i.e., Fc-PEG $250-\mathrm{NHS}, \mathrm{Fc}_{\mathrm{C}} \mathrm{PEG}_{3 \mathrm{k}}-\mathrm{NHS}, \mathrm{Fc}_{\mathrm{C}} \mathrm{PEG}_{5 \mathrm{k}}-\mathrm{NHS}$, and Fc-PEG $10 \mathrm{k}-\mathrm{NHS}$, respectively) were separately grafted onto gold electrodes that were pretreated with cystamine. ${ }^{15,16}$ The PEGs (with the exception of $\mathrm{PEG}_{250}$ ) used here were reported to have a polydispersity index of 1.08 , and their average molecular weights correspond to fully extended lengths of $L=$ 2.1, 27, 40, and $79 \mathrm{~nm}$ (Table 1) for $\mathrm{PEG}_{250}, \mathrm{PEG}_{3 \mathrm{k}}, \mathrm{PEG}_{5 \mathrm{k}}$, and $\mathrm{PEG}_{10 \mathrm{k}}$, respectively. The conformation of the polymers is dictated by the surface density (Figure 1a). If the density is

Received: June 23, 2017

Revised: September 11, 2017

Published: October 4, 2017 
Table 1. Overview of the Used Polymers, Their Molecular Weights and Standard Deviations Calculated from the Reported Polydispersity (1.08), Calculated Flory Radius, $\boldsymbol{R}_{\mathrm{f}}$, Fully Extended Chain Length $(L)$, and the Calculated Surface Density at Which the Chains Start to Interact and Move into a Loose Brush Regime ${ }^{a}$

$\begin{array}{lccc}M_{\mathrm{n}}(\mathrm{kDa}) & \begin{array}{c}R_{\mathrm{f}} \\ (\mathrm{nm})\end{array} & \begin{array}{c}L \\ (\mathrm{~nm})\end{array} & \begin{array}{c}\text { mushroom to brush transition density } \\ \left(\mathrm{mol} / \mathrm{cm}^{2}\right)\end{array} \\ 0.25^{b} & 1.0 & 2.1 & 1.6 \times 10^{-10} \\ 3.4 \pm 1.0 & 4.7 & 27 & 7.4 \times 10^{-12} \\ 5.0 \pm 1.4 & 6.0 & 40 & 4.6 \times 10^{-12} \\ 10 \pm 2.8 & 9.1 & 79 & 2.0 \times 10^{-12}\end{array}$

${ }^{a}$ For the calculations of these densities, see the Experimental Section). ${ }^{b}$ Polydispersity not reported.

sufficiently low (Table 1 ), the polymers exhibit a mushroom conformation. As the surface density increases and the chains start to interact, the polymers extend outward into the solution and form a loose brush.

Cleaned gold electrodes were modified with cystamine, resulting in a monolayer with free amine groups onto which the Fc-PEG-NHS polymers were grafted via their succinimide groups. Cyclic voltammetry measurements of the modified electrodes were performed in a $1 \mathrm{M} \mathrm{NaClO}_{4}$ solution. The results (see Figure 2a for a cyclic voltammogram of a the Fc$\mathrm{PEG}_{250}$ layer) were typical for surface-attached species, with a small peak separation $(<59 \mathrm{mV})$ and a peak current that was linearly dependent on the scan rate, indicating that all the redox couples have sufficient time to contribute to the electron transfer. In this regime, the charge determined from the peak area remains constant independent of scan rate, and the surface density $(\Gamma)$ of the PEG molecules can be determined according to eq 1 .

$$
\Gamma=Q / n F A
$$

In eq $1, Q$ is the charge of the integrated peak area, $A$ the microscopic surface area as determined from sulfuric acid cleaning scans, $F$ the Faraday constant, and $n$ the number of electrons involved ( $n=1$ for ferrocene). At scan rates above $200 \mathrm{~V} / \mathrm{s}$ a significant peak separation became apparent for Fc$\mathrm{PEG}_{250}$, resulting in a characteristic trumpet plot (Figure $2 \mathrm{~b}$ ) when plotting the peak separation, $\eta$, versus the logarithm of the scan rate, $\nu$.

When the peak separation increased past $200 \mathrm{mV}$ and the electron transfer became electrochemically irreversible, the peak potentials changed linearly with the logarithm of the scan rate. In that case, a standard rate constant, $k$, can be determined using Laviron's formulation (eq 2). ${ }^{20}$
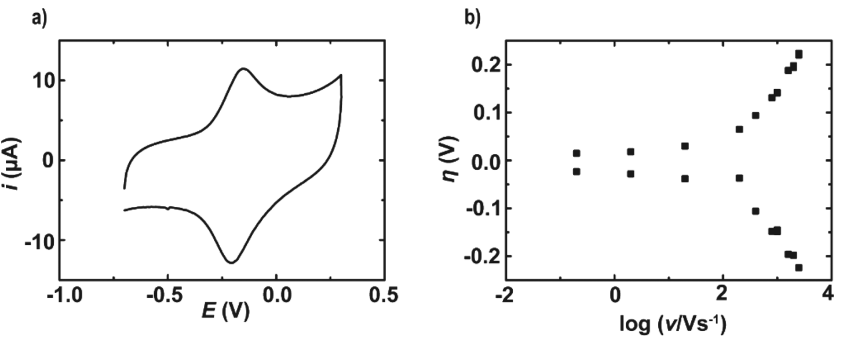

Figure 2. (a) Cyclic voltammogram of a $\mathrm{Fc}_{\mathrm{c}} \mathrm{PEG}_{250}$ monolayer, obtained upon a reaction time of $5 \mathrm{~min}$, recorded in $1 \mathrm{M} \mathrm{NaClO}_{4}$ at 2 $\mathrm{V} / \mathrm{s}$ vs a $\mathrm{Hg} / \mathrm{Hg}_{2} \mathrm{SO}_{4}$ reference electrode. (b) Trumpet plot of the peak separation, $\eta$, versus the logarithm of the scan rate, $\nu$.

$$
k=\frac{\alpha n F \nu_{c}}{R T}=\frac{(1-\alpha) n F \nu_{\mathrm{a}}}{R T}
$$

In eq 2, $\alpha$ is the transfer coefficient, taken as 0.5 , and $R$ and $T$ are the ideal gas constant and the temperature, respectively, while $\nu_{\mathrm{c}}$ and $\nu_{\mathrm{a}}$ are the cathodic and anodic scan rates, respectively. From this treatment, rate constants $k$ of $2.4 \times 10^{3}$ $\mathrm{s}^{-1}$ and $1.7 \times 10^{3} \mathrm{~s}^{-1}$ were found for the anodic and cathodic processes, respectively. These values are significantly larger than the standard rate constants determined for ferrocene moieties on well packed alkane monolayers of similar length, ${ }^{21}$ thus indicating a loosely packed layer in the case of PEG layers. This is confirmed by the measured surface densities, for which values between 2 and $3 \times 10^{-10} \mathrm{~mol} / \mathrm{cm}^{2}$ were obtained when using a surface functionalization time of $5 \mathrm{~min}$.

The peak separation for the longer PEGs was less pronounced and was mostly caused by a shift of the cathodic peak and a smaller shift of the anodic peak combined with significant peak broadening (Figure 3a and d). At increased scan rates, the current became linear with the square root of the scan rate (Figure 3c) following the Randles-Sevcik equation, ${ }^{22}$ indicating an additional dependence of the electron transfer rate on the diffusion of the ferrocene head groups to and from the surface. Since the effect of diffusion on the electron transfer excludes the use of the Laviron method for the determination of rate constants, these were determined using the Nicholson method for diffusing species adjusted for surface-confined species. ${ }^{18,23,24}$ In order to do this, the diffusion constants of ferrocene and oxidized ferrocenium species $\left(D_{\mathrm{Fc}}\right.$ and $D_{\mathrm{Fc}^{+}}$, respectively) were determined from the anodic and cathodic peak currents using the Randles-Sevcik equation (eq 3).

$$
i_{\mathrm{p}}=0.4463 n F A C\left(\frac{n F \nu D}{R T}\right)^{1 / 2}
$$
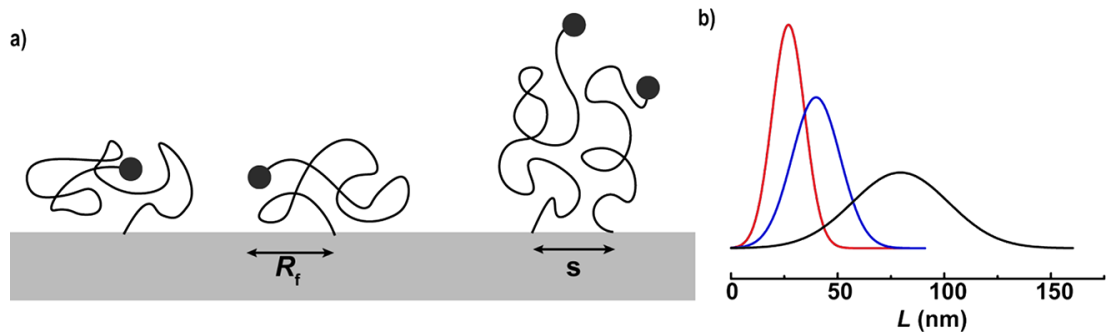

Figure 1. (a) Surface density effect on polymer conformation. If the surface density is sufficiently low, i.e., when the Flory radius $\left(R_{\mathrm{f}}\right)$ is smaller than the distance between the grafting points $(s)$, the polymers are in the mushroom conformation (left). When the surface density is increased, the polymers start to overlap, and a loose brush is formed (right). (b) Gaussian fully extended chain length distribution for the $\mathrm{PEG}_{3 \mathrm{k}} \mathrm{PEG}_{5 \mathrm{k}}$ and $\mathrm{PEG}_{10 \mathrm{k}}$ used here (represented by the colors red, blue, and black, respectively). 

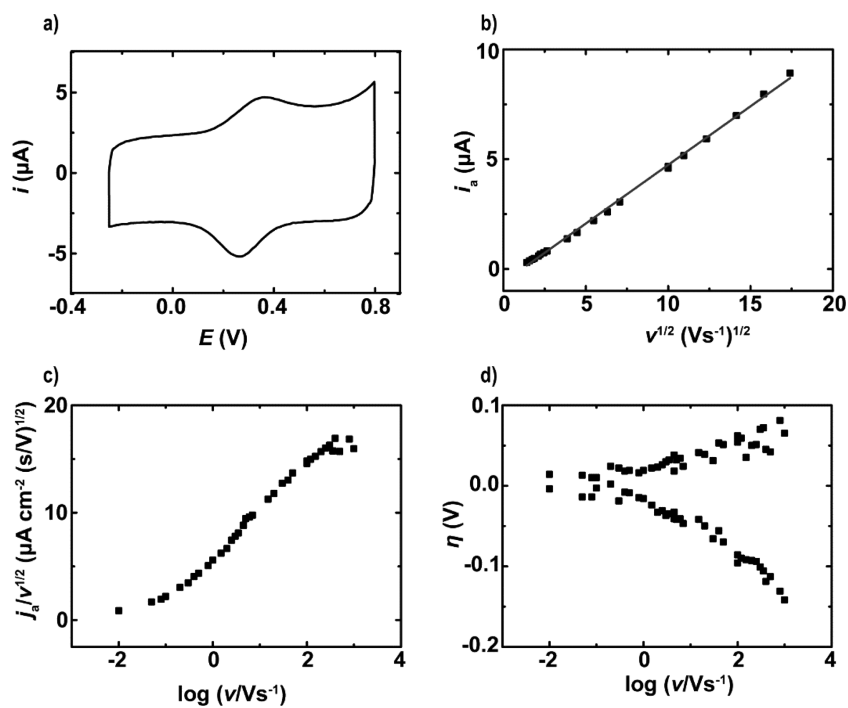

Figure 3. (a) Cyclic voltammogram of a $1.5 \times 10^{-11} \mathrm{~mol} / \mathrm{cm}^{2}$ monolayer of $\mathrm{Fc}_{\mathrm{c}} \mathrm{PEG}_{10 \mathrm{k}}$ (obtained from a reaction time of $90 \mathrm{~min}$ ) in $1 \mathrm{M} \mathrm{NaClO}_{4}$ at $10 \mathrm{~V} / \mathrm{s}$. (b) Anodic peak current, $i$, plotted versus the square root of the scan rate $\nu$; the line is a linear fit to the data. (c) Anodic peak current density $\left(j_{\mathrm{a}}=i_{\mathrm{a}} / A\right)$, normalized to the square root of the scan rate, vs the logarithm of the scan rate. (d) Peak separation vs the logarithm of the scan rate.

In eq 3 , the concentration, $C$, can be replaced by $\Gamma / e$, with $e$ being the layer thickness. Normalizing the anodic peak current densities $\left(j_{\mathrm{a}}=i_{\mathrm{a}} / A\right)$ to $\nu^{1 / 2}$ showed that at higher scan rates $j_{\mathrm{a}} /$ $\nu^{1 / 2}$ became independent of the scan rate, thus reaching a plateau (Figure $3 \mathrm{c}$ ). From the height of this plateau the diffusion coefficients $D_{\mathrm{Fc}}$ and $D_{\mathrm{Fc}^{+}}$were determined using the Randles-Sevcik equation for the polymers in the mushroom conformation. When the surface density is sufficiently low so that the polymers on the surface can be assumed to exist in the mushroom conformation (see Table 1) and do not interact with each other, the average thickness of the polymer layer, $e$, can be taken as the Flory radius.

As can be seen in Table 2, the determined diffusion coefficients show that the bound ferrocenium cation diffuses

Table 2. Diffusion Coefficients and Homogeneous Electron Transfer Rate Constants Determined for Fc-PEG $3 \mathrm{k}$, FcPEG $_{5 \mathrm{k}}$, and Fc-PEG ${ }_{10 \mathrm{k}}$ in the Mushroom Regime

\begin{tabular}{cccc} 
polymer & $D_{\mathrm{Fc}}\left(\times 10^{-12} \mathrm{~cm}^{2} / \mathrm{s}\right)$ & $D_{\mathrm{Fc}^{+}}\left(\times 10^{-12} \mathrm{~cm}^{2} / \mathrm{s}\right)$ & $k_{0}\left(\mathrm{~s}^{-1}\right)$ \\
$\mathrm{Fc}-\mathrm{PEG}_{3 \mathrm{k}}$ & $1.4 \pm 1.0$ & $6.0 \pm 1.3$ & $88 \pm 23$ \\
$\mathrm{Fc}_{\mathrm{PEG}}$ & $1.9 \pm 0.4$ & $6.2 \pm 0.4$ & $114 \pm 104$ \\
$\mathrm{Fc}_{5 \mathrm{k}}$ & $9.1 \pm 6.3$ & $29.8 \pm 9.7$ & $228 \pm 54$ \\
\hline
\end{tabular}

faster (2.5-4 times) than the reduced ferrocene, which is also visible from the peak asymmetry (Figure 3a), the cathodic peak being sharper than the anodic peak. ${ }^{15}$ This effect is not present for ferrocene species in solution ${ }^{25,26}$ but has been noted before for surface-bound Fc-PEG, and has been attributed to the positively charged $\mathrm{Fc}^{+}$being "propelled away" from the surface, caused by electrostatic repulsion between the surface and the $\mathrm{Fc}^{+}$moiety directly after the oxidation step. ${ }^{15}$

The diffusion coefficients increased with increasing polymer length, which is counterintuitive. For the mushroom configuration, the concentration of ferrocene can be represented by a Gaussian distribution with the highest concentration close to the surface. The longer molecules have a higher local density due to the amount of polymer surrounding the ferrocene head, which is expected to slow down diffusion. Indeed, the opposite trend has been observed for different lengths of Fc-modified PNA strands tethered on electrodes, with the diffusion coefficients decreasing and the system becoming more surface confined as the strand length decreased. ${ }^{18}$ On the other hand, it has been shown that the diffusion coefficient for tethered $\mathrm{PEG}_{3 \mathrm{k}}$ is higher than for $\mathrm{PEG}_{600}$ in dichloromethane, even with the $P G_{3 k}$ being in a loose brush conformation and the $P G_{6 k}$ in a mushroom conformation, which has been attributed to an increased influence of the spring constant on the diffusion coefficient. $^{15}$

As mentioned above, using the diffusion coefficients, the homogeneous electron transfer rate constants could be determined using the Nicholson method for diffusing species when the peak separation is between 61 and $212 \mathrm{mV}$. In short, the peak separation is related by a kinetic parameter $\psi$, from which the rate constant can be calculated. ${ }^{23}$ As can be seen in Table 2, the determined rate constants are lower than the value (approximately $2 \times 10^{3} \mathrm{~s}^{-1}$ ) determined for $\mathrm{PEG}_{250}$, in which case the electron transfer is not affected by diffusion. Furthermore, the rate constants increased with increasing polymer length, which could indicate that for the longer molecules the ferrocene end groups are on average closer to the surface. $^{18}$

As the surface density was increased by employing longer reaction times of Fc-PEG-NHS on the cystamine surfaces (Figure $4 \mathrm{a}$ ), the average distance between the anchoring points
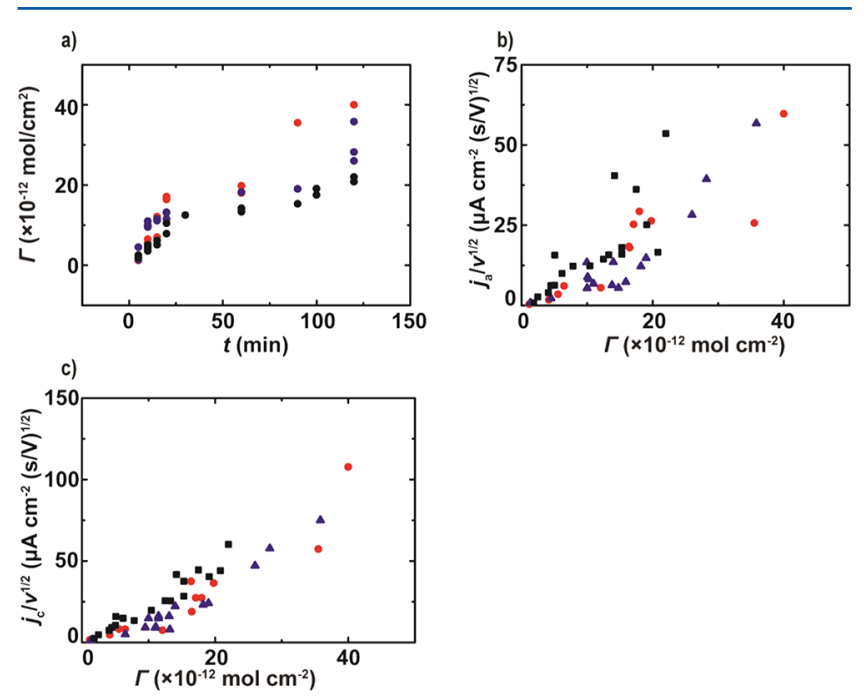

Figure 4. (a) Obtained surface densities as a function of the reaction time, $t .(b, c)$ Anodic $\left(j_{a}\right)$ and cathodic $\left(j_{c}\right)$ peak current densities normalized to the square root of the scan rate, vs surface density, for

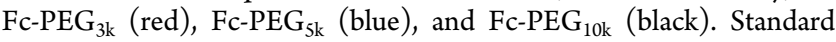
deviations of the values were determined using the least-squares method and fall within a range of $15 \%$.

became smaller than the Flory radius, and consequently the polymer conformation moved from a mushroom into a loose brush regime. In the brush regime, the polymer chains stretch outward into the solution; therefore, the layer thickness $(e)$ is no longer related to the Flory radius, but instead becomes proportional to the average number, $N$, of monomers per chain, by $e \sim p N a \sigma^{1 / 3}$, where $a$ is the monomer size, $\sigma$ the graft density, and $p$ a proportionality coefficient. Since the actual 
layer thickness is unknown in this case, the diffusion and rate constants cannot be directly determined.

The anodic and cathodic peak current densities normalized to the square root of $\nu, j_{\mathrm{c}} / \nu^{1 / 2}$, showed a linear increase upon increasing surface density (Figure $4 \mathrm{~b}$ and $\mathrm{c}$ ). Since the slope of this line is now solely dependent on the parameter $D_{\mathrm{Fc}^{+}}{ }^{1 / 2} / e$ (see eq 3), this parameter was determined for all three PEG lengths in the brush conformation. The values for $D_{\mathrm{Fc}^{+}}{ }^{1 / 2} / e$ were found to be $10.6 \pm 1.7,9.2 \pm 0.6$, and $9.1 \pm 0.8 \mathrm{~s}^{-1 / 2}$ for

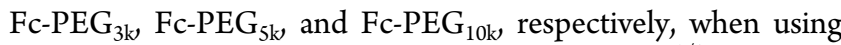
the cathodic peak current densities. The $D_{\mathrm{Fc}}^{1 / 2} / e$ values, determined from the anodic peak current densities, were again lower, $4.5 \pm 1.4,6.6 \pm 0.7$, and $6.1 \pm 1.5 \mathrm{~s}^{-1 / 2}$. The similarity between the PEGs can partly be attributed to the broad size distributions, which show that a large proportion of the size distributions overlap (Figure 1b), despite the difference in $M_{n}$ and a relatively small polydispersity of 1.08 . The fact that the $D^{1 / 2} / e$ values remain constant implies that, as the layer thickness increases, the diffusion constants must increase as well. An increase of the diffusion constant with increased surface density can be explained with an increased proximity of the redox centers to the surface. ${ }^{27}$ For a surface density of $1.5 \times$ $10^{-12} \mathrm{~mol} / \mathrm{cm}^{2}$, and a layer thickness of $40 \mathrm{~nm}$ (half of the fully extended length of $\mathrm{PEG}_{10 \mathrm{k}}$ ), the ferrocene concentration inside that layer can be calculated to be $3.8 \mathrm{mM}$, which is of the same order of magnitude as electrochemical mediators in solution. ${ }^{28}$ This cannot be the sole contribution to the diffusion process, since the diffusion constant is greater for $\mathrm{PEG}_{10 \mathrm{k}}$, which must mean that the local ferrocene concentration is lower than for shorter PEGs, as the layer thickness is larger.

\section{CONCLUSIONS}

Surfaces were modified with different lengths of poly(ethylene glycol) (PEG) end-tethered with a ferrocene moiety and studied using cyclic voltammetry. The shortest Fc-PEG, Fc$\mathrm{PEG}_{250}$, behaved as a surface-confined layer for which homogeneous rate constants could be determined using the Laviron method. These results suggest that a loosely packed layer was formed. For longer Fc-PEGs, diffusion coefficients and homogeneous electron transfer rate constants could be determined when the layers were present in the mushroom conformation, both of which increased with the polymer length. As the surface density was increased, and the polymer layers entered the loose brush regime, the determination of these parameters was not possible due to the unknown layer thickness. Instead, $D^{1 / 2} / e$ values were determined for both ferrocene and ferrocenium, and were shown to be constant for increasing surface densities, implying an increase in diffusion coefficient with surface density. This increase is attributed to an increased chance of electron hopping as the local ferrocene concentration increases. However, the diffusion coefficient also appears to become larger for longer PEGs, while these form a thicker layer and thus a lower Fc concentration. More information is needed to fully explain this increase. Overall, the data presented here highlight that redox-modified polymers attached to an electrode surface show a rich and complex electrochemical behavior, that leads to currents that depend on a multitude of parameters. Unraveling these dependencies will assist in the development of electrochemical sensors that rely on redox behavior of surface-tethered probe molecules.

\section{EXPERIMENTAL SECTION}

Materials. Reagents and solvents were purchased from SigmaAldrich, high-purity water (Milli-Q) was used (Millipore, $R=18.2 \Omega$ ). All bis-NHS-functionalized PEGs were purchased from Nanocs and have a reported dispersity of 1.08 . The Fc-PEG-NHS molecules were synthesized according to known procedures. ${ }^{15} \mathrm{Fc}_{\mathrm{c}} \mathrm{PEG}_{250}-\mathrm{NHS}$ was further purified by column chromatography with dichloromethane as eluent. After the first fraction was removed, the eluent was changed to

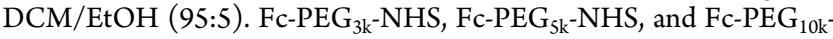
NHS were further purified by size exclusion chromatography (Biobeads SX-1) with DCM as eluent.

2-Ferrocene-Ethylamine. 2-Ferrocene-ethylamine was synthesized by the reduction of ferrocene acetonitrile by $\mathrm{LiAlH}_{4}$ as described in the literature, ${ }^{19}$ with some modifications. An amount of $0.25 \mathrm{~g}(6.5$ mmol) $\mathrm{LiAlH}_{4}$ and $0.6 \mathrm{~g}(4.5 \mathrm{mmol}) \mathrm{AlCl}_{3}$ were carefully added to 10 $\mathrm{mL}$ dry THF while stirring in an ice bath. Ferrocene acetonitrile $(0.5 \mathrm{~g}$, $2.25 \mathrm{mmol}$ ) was dissolved in $5 \mathrm{~mL}$ dry THF and subsequently added to the cooled mixture and refluxed overnight under an argon atmosphere. After cooling, water was added dropwise to decompose the excess $\mathrm{LiAlH}_{4}$. A volume of $0.25 \mathrm{~mL}$ of concentrated $\mathrm{NaOH}$ was added to destroy the formed $\mathrm{AlCl}_{3} / 2$-ferrocene-ethylamine complex. The aqueous phase was extracted three times with diethyl ether. The combined organic phases were dried with $\mathrm{MgSO}_{4}$ and filtered, and the solvent was removed by rotary evaporation. The product was purified by column chromatography with dichloromethane as the eluent. After drying in vacuo, a brown solid was obtained $(0.13 \mathrm{~g} ; 26 \%) .{ }^{1} \mathrm{H}$ NMR $\left(300 \mathrm{MHz}, \mathrm{CDCl}_{3}\right): \delta(\mathrm{ppm}) 4.2(\mathrm{~m}, 9 \mathrm{H}, \mathrm{Fc}), 2.82\left(\mathrm{t}, 2 \mathrm{H}, \mathrm{CH}_{2}-\mathrm{Fc}\right)$, $2.48\left(\mathrm{t}, 2 \mathrm{H}, \mathrm{CH}_{2}-\mathrm{N}\right)$.

$\mathrm{PEG}_{250}$-(NHS)$)_{2}$. The bis-NHS ester of the $\mathrm{PEG}_{250}$ diacid was prepared according to a procedure described earlier. ${ }^{15}$ An amount of $0.44 \mathrm{~g}(3.8 \mathrm{mmol})$ of $\mathrm{N}$-hydroxysuccinimide (NHS) and $0.78 \mathrm{~g}(3.8$ $\mathrm{mmol}$ ) dicyclohexylcarbodiimide (DCC) were added to a stirred solution of $0.4 \mathrm{~g}(1.6 \mathrm{mmol})$ poly(ethylene glycol) bis(carboxymethyl) ether in $30 \mathrm{~mL}$ 1,4-dioxane. After stirring overnight at room temperature under an argon atmosphere, the mixture was filtered to remove the 1,3-dicyclohexylurea precipitate. The solvent was removed by rotary evaporation and the residue was further dried under vacuum. ${ }^{1} \mathrm{H}$ NMR $\left(300 \mathrm{MHz}, \mathrm{CDCl}_{3}\right): \delta(\mathrm{ppm})=4.56\left(\mathrm{~s}, 4 \mathrm{H}, \mathrm{CH}_{2} \mathrm{C}=\mathrm{O}\right)$, $366\left(\mathrm{~s}, 12 \mathrm{H}, \mathrm{C}_{2} \mathrm{H}_{4}-\mathrm{O}\right), 2.81$ (s, $\left.8 \mathrm{H}, \mathrm{NHS}\right)$.

Surface Functionalization and Electrochemistry. All electrochemical measurements were performed on a $\mathrm{CH}$ Instruments bipotentiostat 760D. Cyclic voltammetry measurements were performed in a three-electrode setup using 2-mm-diameter $(\mathrm{CH}$ Instruments) or $1.6-\mathrm{mm}$-diameter (BASi) gold disk electrodes, a platinum wire as the counter electrode, and a $\mathrm{Ag} / \mathrm{AgCl}$ reference electrode in aqueous $1 \mathrm{M} \mathrm{NaClO}_{4}$.

Before modification the electrodes were polished using $50 \mathrm{~nm}$ alumina particles ( $\mathrm{CH}$ Instruments), followed by extensive rinsing with ethanol and $5 \mathrm{~min}$ of ultrasonic treatment in ethanol and $5 \mathrm{~min}$ in Milli-Q water. Subsequently, the electrodes were cleaned electrochemically in $0.5 \mathrm{M} \mathrm{H}_{2} \mathrm{SO}_{4}$ by applying an oxidizing potential of $2 \mathrm{~V}$ for $5 \mathrm{~s}$ followed by a reducing potential of $-0.35 \mathrm{~V}$ for $10 \mathrm{~s}$. Then, the electrode potential was scanned from -0.25 to $1.55 \mathrm{~V}$ and back for 40 cycles at a scan rate of $100 \mathrm{mV} / \mathrm{s}$, the surface area of the electrodes was determined from the $\mathrm{Au}-\mathrm{O}$ reduction peak, using a value of $386 \mu \mathrm{C} /$ $\mathrm{cm}^{2}$ to convert the charge into surface area. ${ }^{29,30}$ The determined surface areas are typically between 1.5 and 2 times the geometrical surface area.

Following a procedure published before, ${ }^{15,16}$ the cleaned electrodes were rinsed with Milli-Q water and ethanol and dried under a flow of $\mathrm{N}_{2}$ and placed immediately in a $2 \mathrm{mM}$ cystamine solution and left overnight. Subsequently, the electrodes were rinsed with Milli- $Q$ water and dried under a flow of $\mathrm{N}_{2}$ and placed in a $0.2 \mathrm{mM} \mathrm{Fc-PEG-NHS}$ solution. The surface densities were varied by changing the reaction time between 5 and $120 \mathrm{~min}$. The prepared electrodes were copiously rinsed with Milli- $Q$ water and subsequently cycled electrochemically at $100 \mathrm{mV} / \mathrm{s}$ in $1 \mathrm{M} \mathrm{NaClO}_{4}$ until a stable signal was obtained. Surface densities were determined from the average charges of at least 3 cyclic voltammograms recorded at scan rates below $500 \mathrm{mV} / \mathrm{s}$, the surface 
densities have an error margin of $<10 \%$. The slope of the currents vs $\nu^{1 / 2}$ were determined over a range of at least 15 different scan rates, standard deviations were determined using the least-squares method and all determined standard deviations are smaller than $15 \%$. The $i R$ compensation function of the bipotentiostat was used to check the effect of solution resistance and double layer capacitance on the peak currents and peak separations at high scan rates, and there was shown to be no difference in the used scan rate regime.

Calculations. The Flory radii $\left(R_{\mathrm{f}}\right)$ in Table 1 were calculated with $R_{\mathrm{f}}=a N^{3 / 5}$ where $a$ is the size of the individual ethylene glycol monomer $\left(a=0.35 \mathrm{~nm}^{31}\right)$ and $N$ the degree of polymerization $(N=6$, 77, 114, and 227 for $\mathrm{PEG}_{250}, \mathrm{PEG}_{3 \mathrm{k}}, \mathrm{PEG}_{5 \mathrm{k}}$ and $\mathrm{PEG}_{10 \mathrm{k}}$, respectively). When the distance between the grafting points (s) of the PEG molecules approaches the $R_{\mathrm{f}}$, the chains start to overlap and form a polymer brush at the critical dimensionless coverage $\sigma_{\text {critical }}=\left(a / R_{\mathrm{f}}\right)^{2}=$ $\left(N_{\mathrm{a}} \Gamma\right) a^{2}$, where $N_{\mathrm{a}}$ is Avogadro's constant. From this the critical surface densities as shown in Table 1 can be determined. ${ }^{14}$

\section{ASSOCIATED CONTENT}

\section{S Supporting Information}

The Supporting Information is available free of charge on the ACS Publications website at DOI: 10.1021/acs.langmuir.7b02160.

Key to the files in the zip folder (PDF)

Cyclic voltammograms (ZIP)

\section{AUTHOR INFORMATION}

\section{Corresponding Authors}

*E-mail: p.jonkheijm@utwente.nl.

*E-mail: j.huskens@utwente.nl.

\section{ORCID}

Pascal Jonkheijm: 0000-0001-6271-0049

Jurriaan Huskens: 0000-0002-4596-9179

\section{Notes}

The authors declare no competing financial interest.

\section{ACKNOWLEDGMENTS}

European Research Council (ERC; eLab4Life project) is acknowledged for funding.

\section{REFERENCES}

(1) Fan, C.; Plaxco, K. W.; Heeger, A. J. Electrochemical Interrogation of Conformational Changes as a Reagentless Method for the Sequence-Specific Detection of DNA. Proc. Natl. Acad. Sci. U. S. A. 2003, 100, 9134-9137.

(2) Prieto-Simón, B.; Campàs, M.; Marty, J. L. Electrochemical Aptamer-Based Sensors. Bioanalytical Reviews 2010, 1, 141-157.

(3) Ricci, F.; Plaxco, K. W. E-DNA Sensors for Convenient, LabelFree Electrochemical Detection of Hybridization. Microchim. Acta 2008, 163, 149-155.

(4) Xiang, Y.; Qian, X.; Jiang, B.; Chai, Y.; Yuan, R. An AptamerBased Signal-on and Multiplexed Sensing Platform for One-Spot Simultaneous Electronic Detection of Proteins and Small Molecules. Chem. Commun. 2011, 47, 4733-4735.

(5) Xiao, Y.; Qu, X.; Plaxco, K. W.; Heeger, A. J. Label-Free Electrochemical Detection of DNA in Blood Serum Via TargetInduced Resolution of an Electrode-Bound DNA Pseudoknot. J. Am. Chem. Soc. 2007, 129, 11896-11897.

(6) Xu, X.; Li, B.; Xie, X.; Li, X.; Shen, L.; Shao, Y. An I-DNA Based Electrochemical Sensor for Proton Detection. Talanta 2010, 82, $1122-1125$

(7) Zhang, K.; Zhu, X.; Wang, J.; Xu, L.; Li, G. Strategy to Fabricate an Electrochemical Aptasensor: Application to the Assay of Adenosine Deaminase Activity. Anal. Chem. 2010, 82, 3207-3211.
(8) De Gennes, P. G. Conformations of Polymers Attached to an Interface. Macromolecules 1980, 13, 1069-1075.

(9) Alexander, S. Adsorption of Chain Molecules with a Polar Head. A Scaling Description. J. Phys. (Paris) 1977, 38, 983-987.

(10) Pépin, M. P.; Whitmore, M. D. Monte Carlo and Numerical Self-Consistent Field Study of End-Tethered Polymers in Good Solvent. J. Chem. Phys. 1999, 111, 10381-10388.

(11) Karaiskos, E.; Bitsanis, I. A.; Anastasiadis, S. H. Monte Carlo Studies of Tethered Chains. J. Polym. Sci., Part B: Polym. Phys. 2009, 47, 2449-2461.

(12) Wu, T.; Efimenko, K.; Genzer, J. Combinatorial Study of the Mushroom-to-Brush Crossover in Surface Anchored Polyacrylamide. J. Am. Chem. Soc. 2002, 124, 9394-9395.

(13) Dumont, E. L. P.; Belmas, H.; Hess, H. Observing the Mushroom-to-Brush Transition for Kinesin Proteins. Langmuir 2013, 29, $15142-15145$.

(14) Abbou, J.; Anne, A.; Demaille, C. Probing the Structure and Dynamics of End-Grafted Flexible Polymer Chain Layers by Combined Atomic Force-Electrochemical Microscopy. Cyclic Voltammetry within Nanometer-Thick Macromolecular Poly(Ethylene Glycol) Layers. J. Am. Chem. Soc. 2004, 126, 10095-10108.

(15) Anne, A.; Moiroux, J. Quantitative Characterization of the Flexibility of Poly(Ethylene Glycol) Chains Attached to a Glassy Carbon Electrode. Macromolecules 1999, 32, 5829-5835.

(16) Anne, A.; Demaille, C.; Moiroux, J. Terminal Attachment of Polyethylene Glycol (Peg) Chains to a Gold Electrode Surface. Cyclic Voltammetry Applied to the Quantitative Characterization of the Flexibility of the Attached Peg Chains and of Their Penetration by Mobile Peg Chains. Macromolecules 2002, 35, 5578-5586.

(17) Abbou, J.; Anne, A.; Demaille, C. Accessing the Dynamics of End-Grafted Flexible Polymer Chains by Atomic Force-Electrochemical Microscopy. Theoretical Modeling of the Approach Curves by the Elastic Bounded Diffusion Model and Monte Carlo Simulations. Evidence for Compression-Induced Lateral Chain Escape. J. Phys. Chem. B 2006, 110, 22664-22675.

(18) Hüsken, N.; Gẹbala, M.; La Mantia, F.; Schuhmann, W.; Metzler-Nolte, N. Mechanistic Studies of Fc-Pna(-DNA) Surface Dynamics Based on the Kinetics of Electron-Transfer Processes. Chem. - Eur. J. 2011, 17, 9678-9690.

(19) Seiwert, B.; Karst, U. Analysis of Cysteine-Containing Proteins Using Precolumn Derivatization with N-(2-Ferroceneethyl)Maleimide and Liquid Chromatography/Electrochemistry/ Mass Spectrometry. Anal. Bioanal. Chem. 2007, 388, 1633-1642.

(20) Laviron, E. General Expression of the Linear Potential Sweep Voltammogram in the Case of Diffusionless Electrochemical Systems. J. Electroanal. Chem. Interfacial Electrochem. 1979, 101, 19-28.

(21) Chidsey, C. E. D. Free Energy and Temperature Dependence of Electron Transfer at the Metal-Electrolyte Interface. Science 1991, 251, 919-922.

(22) Bard, A. J.; Faulkner, L. R. Electrochemical Methods: Fundamentals and Applications, 2nd ed.; John Wiley \& Sons: New York, 2001.

(23) Nicholson, R. S. Theory and Application of Cyclic Voltammetry for Measurement of Electrode Reaction Kinetics. Anal. Chem. 1965, 37, $1351-1355$.

(24) Anne, A.; Demaille, C. Dynamics of Electron Transport by Elastic Bending of Short DNA Duplexes. Experimental Study and Quantitative Modeling of the Cyclic Voltammetric Behavior of 3'Ferrocenyl DNA End-Grafted on Gold. J. Am. Chem. Soc. 2006, 128, $542-557$.

(25) Martin, R. D.; Unwin, P. R. Theory and Experiment for the Substrate Generation/Tip Collection Mode of the Scanning Electrochemical Microscope: Application as an Approach for Measuring the Diffusion Coefficient Ratio of a Redox Couple. Anal. Chem. 1998, 70, 276-284.

(26) Mampallil, D.; Mathwig, K.; Kang, S.; Lemay, S. G. Redox Couples with Unequal Diffusion Coefficients: Effect on Redox Cycling. Anal. Chem. 2013, 85, 6053-6058. 
(27) Akhoury, A.; Bromberg, L.; Hatton, T. A. Interplay of Electron Hopping and Bounded Diffusion During Charge Transport in Redox Polymer Electrodes. J. Phys. Chem. B 2013, 117, 333-342.

(28) Liu, B.; Bard, A. J.; Mirkin, M. V.; Creager, S. E. Electron Transfer at Self-Assembled Monolayers Measured by Scanning Electrochemical Microscopy. J. Am. Chem. Soc. 2004, 126, 1485-1492.

(29) Trasatti, S.; Petrii, O. A. Real Surface Area Measurements in Electrochemistry. J. Electroanal. Chem. 1992, 327, 353-376.

(30) Tremiliosi-Filho, G.; Dall'Antonia, L. H.; Jerkiewicz, G. Limit to Extent of Formation of the Quasi-Two-Dimensional Oxide State on

Au Electrodes. J. Electroanal. Chem. 1997, 422, 149-159.

(31) Allen, C.; Dos Santos, N.; Gallagher, R.; Chiu, G. N. C.; Shu, Y.; Li, W. M.; Johnstone, S. A.; Janoff, A. S.; Mayer, L. D.; Webb, M. S.; Bally, M. B. Controlling the Physical Behavior and Biological Performance of Liposome Formulations through Use of Surface Grafted Poly(Ethylene Glycol). Biosci. Rep. 2002, 22, 225-250. 